\title{
Injectable Hydrogels for Regenerative Medicine
}

\author{
Byung-Soo Kim ${ }^{1} \cdot$ Chong-Su Cho $^{2}$
}

Published online: 11 September 2018

(C) The Korean Tissue Engineering and Regenerative Medicine Society and Springer Science+Business Media B.V., part of Springer Nature 2018

Hydrogels are being used extensively for various biomedical applications such as tissue engineering, regenerative medicine, and drug delivery. Clinically used hydrogels include hyaluronic acid, fibrin, collagen, gelatin, alginate, hydroxyethylcellulose, carboxymethylcellulose, poly (2hydroxyethyl methacrylate), poly (2-hydroxypropyl methacrylate), poly (acrylic acid), and poly (ethylene glycol) [1]. The hydrogel materials have several advantages for biomedical applications. They can mimic the three-dimensional environment of extracellular matrix in natural tissues. Thus, cells and biomolecules housed in the hydrogels can take the advantages of the natural tissue environment to exert the intrinsic function of the cells and biomolecules for tissue regeneration. The hydrogels can be modified to improve biocompatibility, cell adhesiveness, biodegradability, mechanical properties, and gel formation rate of hydrogels [2,3]. In addition, the hydrogels can deliver cells or biomolecules to patients in a minimally invasive way for improving the patient compliance to treatment.

This special issue presents six review articles to overview and update the current technologies of hydrogel and their applications to various fields. The review by Seo and

Byung-Soo Kim

byungskim@snu.ac.kr

$\triangle$ Chong-Su Cho

chocs@snu.ac.kr

1 School of Chemical and Biological Engineering, Seoul National University, 1 Gwanak-ro, Gwanak-gu, Seoul 08826, Korea

2 Research Institute for Agriculture and Life Sciences, Seoul National University, 1 Gwanak-ro, Gwanak-gu, Seoul 08826, Korea his colleagues summarized the electrostatically interactive hydrogels produced through the electrostatic interactions between anionic and cationic polyelectrolytes for drug delivery applications. The hydrogels have advantages of easy preparation and reversible gel forming. Patel et al. overviewed polypeptide thermogels for stem cell therapy applications because the polypeptide thermogels could provide cell adhesion sites and employ mild physiological conditions for cell incorporation. The polypeptides with desirable functional groups can be selected for induction of differentiation of stem cells housed in the polypeptide thermogels. A review by Gopinathan and Noh described hydrogel preparation by various methods of click chemistry and applications to tissue engineering and three-dimensional bioprinting. The click chemistry does not use toxic solvents and allows for instant formation of hydrogels, enabling to retain live cells or biomolecules in the resultant hydrogels and allowing hydrogels to be printed. The review by Park and his colleague summarized the recent advancement in in situ cross-linkable hydrogels and their applications to tissue regeneration. Cho et al. discussed the current status of hydrogels, which are used alone or in combination with stem cells and growth factors, for applications in plastic and reconstructive surgery. The last review, contributed by Sontyana et al., described various biopolymeric hydrogel materials and their applications to tissue regeneration using in situ imaging techniques.

This special issue will provide future perspectives of hydrogel technology and may help to develop next-generation hydrogel technologies and expand the biomedical applications of hydrogels. We hope this special issue will contribute to hydrogel technology that continue to advance to benefit human health care. 
Acknowledgements The editors express a deep sense of gratitude to all the invited authors for their valuable efforts for the review articles.

\section{References}

1. Li J, Mooney DJ. Designing hydrogels for controlled drug delivery. Nat Rev Mater. 2016;1:16071.
2. Yang JA, Yeom J, Hwang BW, Hoffman AS, Hahn SK. In situforming injectable hydrogels for regenerative medicine. Prog Polym Sci. 2014;39:1973-86.

3. Buwalda SJ, Boere KW, Dijkstra PJ, Feijen J, Vermonden T, Hennink WE. Hydrogels in a historical perspective: from simple networks to smart materials. J Control Release. 2014;190:254-73. 